\title{
Optical imaging through dynamic turbid media using the Fourier-domain shower-curtain effect: supplementary material
}

\author{
EITAN EDREI AND GIULIANO SCARCELLI* \\ Fischell Department of Bioengineering, University of Maryland, College Park, Maryland, USA \\ *Corresponding author: scarc@umd.edu
}

Published 13 January 2016

\begin{abstract}
This document provides supplementary information to "Optical imaging through dynamic turbid media using the Fourier-domain shower-curtain effect," http://dx.doi.org/10.1364/optica.3.000071. It contains information regarding the optical setup and the data processing as well as a mathematical derivation of the underlying principles used in the main text. In addition, we provide here a characterization of the scattering layers and their dynamics as well as a comparison of this study with other methods in the field and some results which were not included in the main text. @) 2016 Optical Society of America
\end{abstract}

http://dx.doi.org/10.1364/optica.3.000071.s001

\section{Methods}

Optical Setup: We used a He-Ne continuous wave laser of $30 \mathrm{~mW}$ output power, expanded its beam diameter to $8 \mathrm{~mm}$ with a $4.5 \mathrm{x}$ telescope and illuminated a ground glass diffuser (Thorlabs, $220 \mathrm{Grit}$ ). We placed the object apertures $1 \mathrm{~cm}$ after the ground glass diffuser. As objects, we used a USAF resolution target (Thorlabs) or we fabricated custom patterns with standard photolithography masks. We placed the turbid medium of interest (e.g. ground glass, cataract, chicken tissue, paper) at 50 to $200 \mathrm{~mm}$ from the object. Finally, we built an optical system to image the back of the turbid medium using an objective lens (Olympus, 4X DIN, 0.1 NA) and a CMOS camera (Mightex MCE-B013). For image reconstruction, we continuously moved the first diffuser and typically acquired 3000 frames with an integration times of $10 \mathrm{~ms}-100 \mathrm{~ms}$.

Imaging correlography, Principle and Data processing: The following mathematical derivation is adapted to our experimental situation from the general correlography procedure explained in Refs. 18-20 of the main text. By illuminating an object with a speckle pattern, the speckled object field $\boldsymbol{f}_{\boldsymbol{n}}(\boldsymbol{x}, \boldsymbol{y})$ is obtained right after the object (the subscript $\mathrm{n}$ refers to a specific illuminating speckle pattern). In the far field condition, because the speckle illumination is partially coherent, both a coherent term: $\left|\widetilde{\boldsymbol{F}}\left[\boldsymbol{f}_{\boldsymbol{n}}(\boldsymbol{x}, \boldsymbol{y})\right]\right|^{2}$ and a background incoherent term: $\boldsymbol{B}(\boldsymbol{x}, \boldsymbol{y})$ are formed. By averaging many speckle realizations and subtracting the averaged pattern from each recorded image the incoherent term can be eliminated. The inverse Fourier transform of a single camera shot therefore yields the autocorrelation of the speckled object field: $\widetilde{F}^{-1}\left[\left|\widetilde{F}\left[f_{n}(x, y)\right]\right|^{2}\right]=f_{n}(x, y) \star f_{n}(x, y)$. The speckled object field $\boldsymbol{f}_{\boldsymbol{n}}(\boldsymbol{x}, \boldsymbol{y})$ is a convolution of the object shape
$\boldsymbol{O}(\boldsymbol{x}, \boldsymbol{y})$ (taking the value 1 within the object or 0 otherwise) and the PSF of a specific speckle configuration $\boldsymbol{S}_{\boldsymbol{n}}(\boldsymbol{x}, \boldsymbol{y})$. Substituting $\boldsymbol{f}_{\boldsymbol{n}}(\boldsymbol{x}, \boldsymbol{y})=\boldsymbol{O}(\boldsymbol{x}, \boldsymbol{y}) * \boldsymbol{S}_{\boldsymbol{n}}(\boldsymbol{x}, \boldsymbol{y})$ and summing over $\mathrm{N}$ independent realizations of the speckle illumination, the following can be obtained:

$$
\begin{aligned}
& \sum_{n=1}^{N}\left|f_{n}(x, y) \star f_{n}(x, y)\right|^{2}= \\
& \propto|O(x, y)|^{2} \star|O(x, y)|^{2} * \sum_{n=1}^{N}\left|S_{n}(x, y) \star S_{n}(x, y)\right|^{2}
\end{aligned}
$$

For a large number of frames generated from independent speckle configurations the sum yields a smoothed peaked function; therefore the convolution yields the autocorrelation of the object, which is then fed to the phase retrieval algorithm.

The correlography reconstruction process shares similarities with memory-effect based reconstructions and other speckle interferometry work. Two key differences are that (a) the Fourier Transform of the object is obtained coherently; (b) at this stage no turbid medium has been considered yet. The scattering turbid medium is addressed through the shower-curtain effect so that that for every frame the Fourier Transform of the object is directly read on the "curtain".

In the computer algorithm, from each recorded camera pattern, we took the absolute value of the Fourier transform and added up the resulting patterns to get an estimated autocorrelation of the object. We smoothed the computed autocorrelation pattern by applying an optimal threshold on the power spectrum of the reconstructed image and windowed the central portion of the autocorrelation with a Tukey window function. The optimized autocorrelation was then fed to a phase retrieval algorithm for image reconstruction. We used a basic 
version of a Fienup phase retrieval algorithm in which a random phase matrix was generated and the $\beta$ parameter was varied from 2 to 0 in steps of 0.05 (similar to references 2,3 of the main text). We carried 10 iterations for each value of $\beta$. The algorithm was applied several times using different random initial matrixes, and the best reconstruction was chosen and smoothed by a Gaussian filter.

\section{Traditional shower curtain effect resolution}

We evaluated the resolution degradation due to the traditional shower curtain effect experimentally by using a USAF chart and quantifying the smallest resolvable feature corresponding to turbid media of increasing scattering lengths. To vary the turbidity of the

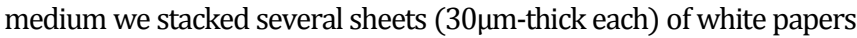
or several layers (50 $\mu \mathrm{m}$ thick each) of translucent tape. The results are presented in Fig. S1. To compute the scattering lengths, we characterized the effective scattering coefficient for both conditions in transmission experiments and obtained $25 \mu \mathrm{m}^{-1}$ (paper) and $200 \mu \mathrm{m}^{-1}$ (tape). Interestingly, the resolution behavior obtained with paper (red) and tape (black) collapse on the same curve. This behavior is also consistent with the results obtained with biological tissue; we were able to resolve $20 \mu \mathrm{m}$ sized features through a $800 \mu \mathrm{m}$ thick chicken breast tissue (i.e. corresponding to $\sim 3$ scattering lengths) $[1,2]$.

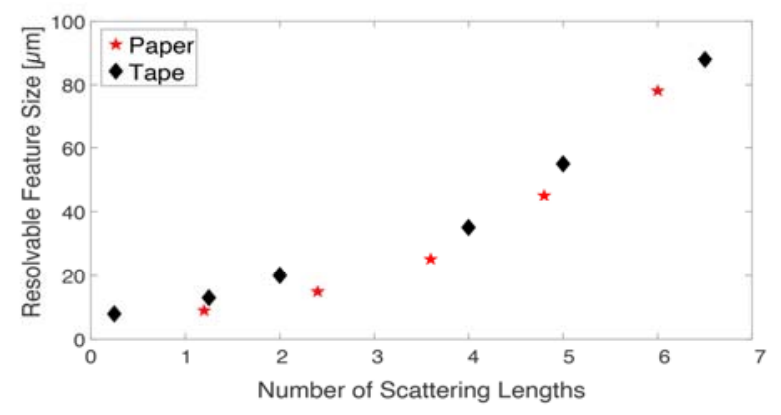

Fig. S1. Degradation of the imaging resolution by the shower curtain effect for increasing layers of tapes (black) and papers (red).

\section{Spatio-temporal behavior of light correlations in shower-curtain effect and memory effect}

To investigate the spatial correlation properties of our protocol in comparison to the memory effect, we measured the memory angular range for increasing number of papers (same as in Fig. S1) by placing the scattering layers on a rotational stage and plotting the decorrelation curve for each number of scattering papers. The angular width of the memory effect was estimated by the FWHM of the decorrelation plot. We plotted the inverse value of this angle versus the resolution obtained for the same scattering medium when placed right in front of the object and found a strong linear correlation (Fig. S2). Therefore, the imaging resolution through the scattering layer via shower curtain effect is inversely proportional to the angular range allowed by memory effect. This result confirms that the near-field spatial correlations between front-side and back-side of the turbid medium that are exploited in the shower-curtain effect are due to the same phenomenon that regulates the far-field spatial correlations exploited in memory effect protocols. For an intuitive understanding, the angular range allowed by memory-effect in a given turbid medium, can be interpreted as the maximum angle (i.e. NA) through which we can see an object with the shower-curtain effect.

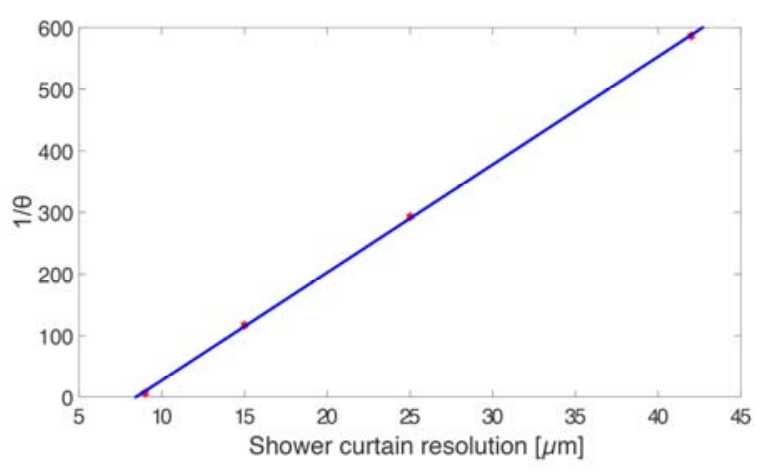

Fig. S2. The resolution determined by the shower-curtain effect is inversely related to the angular width of the memory effect.

On the other hand, the temporal behavior of the light correlation is different in shower-curtain compared to memory-effect methods. Here, we demonstrate that under the condition in which the spatial correlations of the turbid medium are completely lost, the contrast obtained by imaging through the shower-curtain is not affected. To prove this we compared the autocorrelation of a double-slit aperture placed behind the ground glass diffuser in two conditions of static vs rapidly rotating. To obtain the autocorrelation of the double slit, we performed the exact same image processing as shown in Fig. 1 of the main text excluding the phase retrieval process. A line section of the results is plotted in Fig. S3a. The rotation of the ground glass did not introduce any loss of contrast in the autocorrelation domain; in fact, the autocorrelation was better in the dynamic case due to the spatial averaging of different scattering configurations of the rotating turbid medium. Next, we measured the far-field spatial autocorrelations in the static vs dynamic conditions that one would need to reconstruct the object shape in a memory-effect protocol such as Ref. 3 of the main text. As expected, we observed a near complete loss of the spatial correlations as shown in Fig. S3b because the decorrelation time due to the diffuser rotation is close to four orders of magnitude $(<1 \mu \mathrm{s})$ faster than the camera acquisition time $(5 \mathrm{~ms})$. These results emphasize that our procedure is immune to movements of the turbid medium.
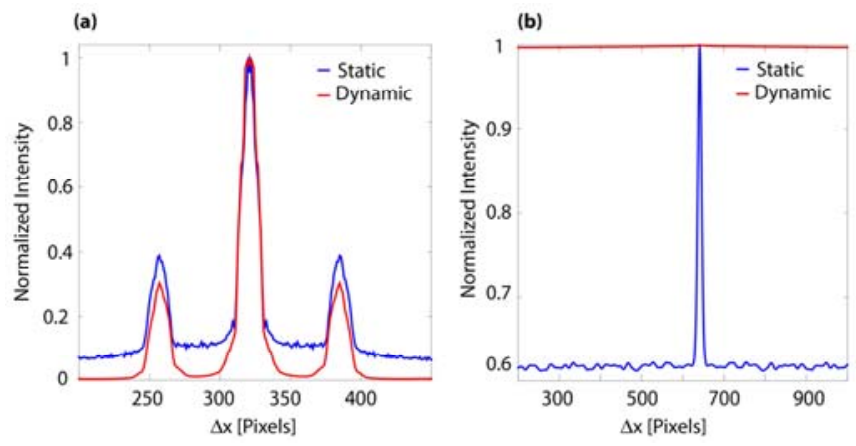

Fig. S3. (a) Cross-section of the autocorrelation of a double slit aperture (obtained by our method described in the main text) when placed behind a static ground glass (blue) and a rotating ground glass (red). (b) Spatial autocorrelations of the pattern recorded after the static ground glass (blue) and the rotating ground glass (red). 


\section{Shortening the far field condition distance with speckle correlography}

The aim of this section is to determine the condition under which the image recorded by the camera is given by the Fourier transform of the field transmitted through the object, i.e: $I(x, y)=\left|\widetilde{\boldsymbol{F}}\left[\boldsymbol{f}_{\boldsymbol{n}}(\boldsymbol{x}, \boldsymbol{y})\right]\right|^{\mathbf{2}}$. For a perfectly coherent beam this will be true when the far field condition $z>2 \frac{D^{2}}{\lambda}$ is satisfied (D being the size of the object). However, for partially coherent light the "far-field" condition is satisfied at shorter distances as we will derive following the formalism of Goodman [3].

Consider a partially coherent light transmitted through an aperture placed in the $\xi, \eta$ plane. The intensity recorded on a camera placed in the $x, y$ plane some distance away can be obtained by propagating the mutual transmitted intensity $\boldsymbol{J}_{t}$ using a Fresnel integral:

$$
I(x, y) \approx \frac{1}{(\lambda z)^{2}} \iint_{-\infty}^{\infty} \iint_{-\infty}^{\infty} \boldsymbol{J}_{t}\left(\xi_{1}, \eta_{1} ; \xi_{2}, \eta_{2}\right) e^{-j \frac{2 \pi}{\lambda}\left(r_{2}^{\prime}-r_{1}^{\prime}\right)} d \xi_{1} d \eta_{1} d \xi_{2} d \eta_{2}
$$

Where $r_{1}^{\prime}, r_{2}^{\prime}$ are the distances between the points in the aperture plane and the observation plane, thus: $r_{2}^{\prime}-r_{1}^{\prime} \approx \frac{1}{2 z}\left[\left(\xi_{2}^{2}+\eta_{2}^{2}\right)-\right.$ $\left.\left(\xi_{1}^{2}+\eta_{1}^{2}\right)-2(x \Delta \xi+y \Delta \eta)\right]$. This can be simplified into: $r_{2}^{\prime}-r_{1}^{\prime} \approx \frac{1}{z}[\bar{\xi} \Delta \xi+\bar{\eta} \Delta \eta-x \Delta \xi-y \Delta \eta]$, where we defined: $\bar{\xi}=\frac{\xi_{1}+\xi_{2}}{2}, \bar{\eta}=\frac{\eta_{1}+\eta_{2}}{2}$.

The recorded intensity can therefore be written as: $I(x, y)$

$\approx \frac{1}{(\lambda z)^{2}} \iint_{-\infty}^{\infty} \iint_{-\infty}^{\infty} \boldsymbol{J}_{t}\left(\xi_{1}, \eta_{1} ; \xi_{2}, \eta_{2}\right) e^{-j \frac{2 \pi}{\lambda z}([\bar{\xi} \Delta \xi+\bar{\eta} \Delta \eta-x \Delta \xi-y \Delta \eta])} d \xi_{1} d \eta_{1} d \xi_{2} d \eta_{2}$

The far-field condition in which the Fresnel integral approaches a Fourier transform is given by: $z \gg \frac{\bar{\xi} \Delta \xi+\bar{\eta} \Delta \eta}{\lambda}$. However, the mutual transmitted intensity function $\boldsymbol{J}_{t}$ is proportional to the complex coherence factor $\mu(\Delta \xi, \Delta \eta)$ which is non-zero only within the coherence area of the illumination beam. Thus, the maximum value of $\bar{\xi}, \bar{\eta}$ is the aperture size $\mathrm{D} / 2$; on the other hand, the maximum value for $\Delta \xi, \Delta \eta$ in which $\mu(\Delta \xi, \Delta \eta)$ has a significant value is the correlation radius $\mathrm{R}_{c} / 2$; therefore, the "far-field" condition reduces to: $z \gg \frac{D R_{c}}{4 \lambda}$. In the main text we further simplified this condition to $z>2 \frac{D R_{C}}{\lambda}$.

We verified experimentally that the far-field condition is greatly shortened as predicted theoretically by the previous derivation. In order to do so we used a double slit aperture (slit width $150 \mu \mathrm{m}$, slit distance $1 \mathrm{~mm}$ ). When the aperture was illuminated with a coherent laser beam the diffraction pattern recorded $50 \mathrm{~mm}$ away showed a clear near-field Fresnel pattern. However, in the same experimental configuration, by changing the illumination of the slits to a speckle pattern and performing our correlography analysis (the turbid medium placed $50 \mathrm{~mm}$ from the aperture), we observed a Fraunhoferlike interference-diffraction pattern. (a)

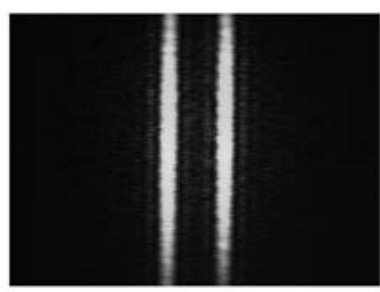

(c)

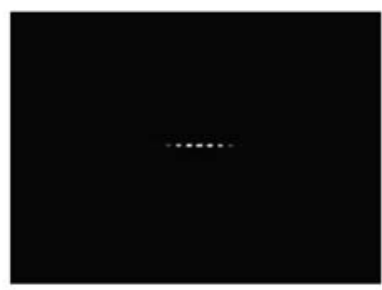

(b)

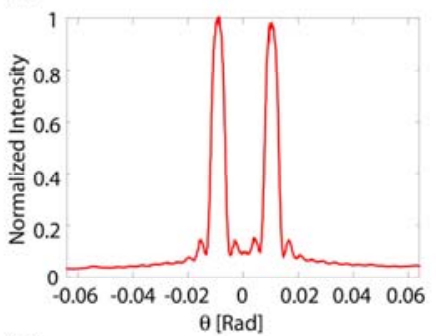

(d)

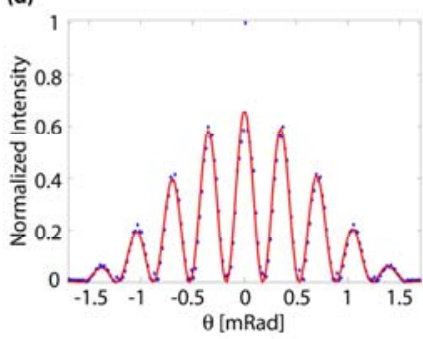

Fig. S4. (a) Diffraction pattern observed $50 \mathrm{~mm}$ from the aperture when illuminated by a coherent laser beam. (b) The line plot corresponding to the average of the recorded pattern shows a clear near-field Fresnel pattern. (c) Diffraction pattern observed $50 \mathrm{~mm}$ from the aperture as retrieved by correlography analysis when the slits are illuminated by a speckle pattern. (d) The corresponding averaged line plot clearly shows a far-field diffraction pattern, as confirmed by the fit to the Fourier transform of the double slit (red line).

\section{Decorrelation measurements}

We characterized the decorrelation times for both the first diffuser used to generate the different speckled configurations in the various experiments (Fig. S5a) as well as the rapidly rotating ground glass used in Fig. 3 of the main text and Fig. S3 (Fig. S5b).

For the decorrelation measurements, a laser beam passed through the scattering layer of interest and a fraction of the generated speckle pattern was continuously recorded at a distant plane using a photodiode (Thorlabs PDA36A). (a)

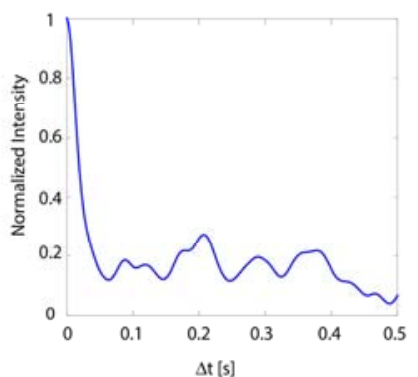

(b)

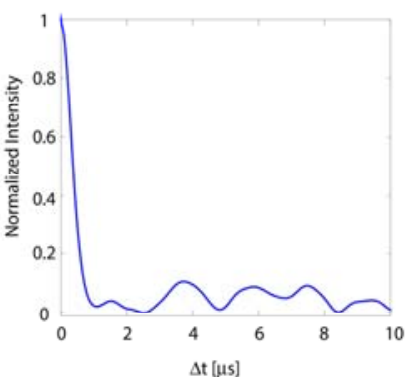

Fig. S5. (a) Spatial decorrelation time of the first diffuser. (b) Spatial decorrelation time of the rapidly rotating ground glass used as turbid medium. 


\section{Imaging through thick tissue}

To demonstrate the feasibility of this method to image through thick scattering layers we placed an object behind a $3.3 \mathrm{~mm}$ thick chicken breast tissue. Using our speckle correlography protocol, we were able to reconstruct an image of the object; we used a similar setup as in Fig. 1 of the main text, but we increased the distance between object and turbid medium to $600 \mathrm{~mm}$ and used a scientific CMOS camera (Andor Neo).
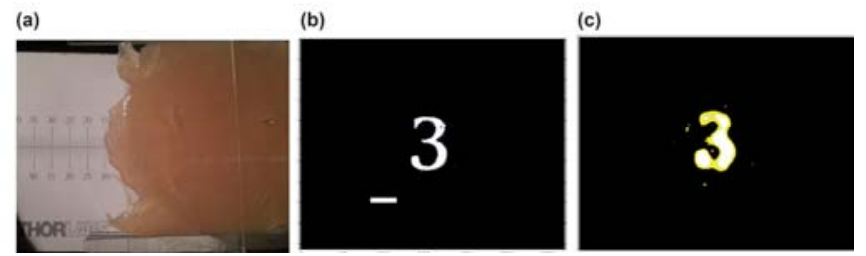

Fig. S6. (a) 3.3-mm thick piece of chicken breast tissue placed $\sim 10 \mathrm{~mm}$ in-front of an object, totally blocks our ability to image it. (b) Object. Scale bar, $100 \mu \mathrm{m}$. (c) Reconstructed image.

\section{References}

1. M. Mesradi, A. Genoux, V. Cuplov, D. A. Haidar, S. Jan, I. Buvat, and F. Pain, "Experimental and analytical comparative study of optical coefficient of fresh and frozen rat tissues," Journal of Biomedical Optics $\mathbf{1 8}$ (2013).

2. J. Bertolotti, E. G. van Putten, C. Blum, A. Lagendijk, W. L. Vos, and A. P. Mosk, "Non-invasive imaging through opaque scattering layers," in Conference on Adaptive Optics and Wavefront Control for Biological Systems(San Francisco, CA, 2015).

3. J. W. Goodman, and R. L. Haupt, Statistical optics (John Wiley \& Sons, 2015) 